\title{
Study on the Release of Muscle Passive Force by Ultrasonic Mechanical Effect and its Related Mechanism
}

\section{Xuebing Yang}

Shaanxi Normal University

\section{Xueqing Zhang}

Shaanxi Normal University

Pan Li

Shaanxi Normal University

Liang Tang

Shaanxi Normal University

Dean Ta

Fudan University

Jianzhong Guo ( $\nabla$ guojz@snnu.edu.cn )

Shaanxi Normal University

\section{Research Article}

Keywords: muscle passive force, pulsed ultrasound, the standard linear solid model, viscosity coefficient, elasticity coefficient, fibril content.

Posted Date: December 28th, 2021

DOl: https://doi.org/10.21203/rs.3.rs-1188396/v1

License: (c) (i) This work is licensed under a Creative Commons Attribution 4.0 International License. Read Full License 
1 Study on the release of muscle passive force by ultrasonic mechanical

2 effect and its related mechanism

Xuebing Yang ${ }^{1}$, Xueqing Zhang ${ }^{1}$, Pan $\mathrm{Li}^{1}$, Liang Tang ${ }^{2}$, Dean Ta ${ }^{3,4,5}$ and Jianzhong Guo ${ }^{1 *}$

${ }^{1}$ School of Physics and Information Technology, Shaanxi Normal University, Xi'an 710062, China

${ }^{2}$ Institute of Sports Biology, Shaanxi Normal University, Xi'an 710119, China

3 Department of Electronic Engineering, Fudan University, Shanghai 200433, China

${ }^{4}$ Human Phenome Institute, Fudan University, Shanghai 201203, China

${ }^{5}$ Key Laboratory of Medical Imaging Computing and Computer Assisted Intervention (MICCAI) of Shanghai, Shanghai 200032, China

* corresponding author: guojz@snnu.edu.cn (J Guo)

\section{Abstract}

Background: Excessive muscle force impedes physical movement and relaxing passive muscle force substantially improves movement impairment. Ultrasound is an energy carrier with the characteristics of repetitive mechanical stimulation, which may be a feasible method to relieve muscle tension.

Methods: We performed stress relaxation experiments on soleus muscle and combine the obtained results with the standard linear solid model to extract information of viscoelastic effect of ultrasound on muscle, and calculated muscle fibril content by histological analysis.

Results: Ultrasound can accelerate muscle stress relaxation; the viscosity and elasticity coefficient of the ultrasound group was higher than that of the control group, and there was no significant difference between the three ultrasound intensities; H\&E 
staining showed that muscle fibrillar content decreased and the matrix substance increased.

Conclusion: We considered that ultrasound can change the microstructure of muscle, and the matrix substance plays a significant role in the relaxation process. In this paper, the relationship between muscle viscoelasticity and passive muscle force is obtained. The results provide an important theoretical basis and a feasible method for monitoring muscle functional characteristics by measuring muscle viscoelasticity.

Keywords: muscle passive force, pulsed ultrasound, the standard linear solid model, viscosity coefficient, elasticity coefficient, fibril content.

\section{Background}

As a muscle is passively stretched, it resists with passive force such as tension, impeding joint movement, reducing muscle flexibility, and increasing the risk of muscle injury [1,2]. Because this significantly affects the level at which an individual can function and perform, it is particularly important for athletes to relieve muscle tension and improve flexibility after exercise.

Proprioceptive neuromuscular facilitation (PNF) and static passive stretching are two most commonly used stretching techniques in athletics and clinical practice [3, 4]. Another method — mechanical vibration that has been shown improve flexibility [5-7]. The release of muscle tension can correct muscle imbalances, improve joint range of motion, relieve muscle soreness and joint pressure, and help maintain physiological muscle length. The most common and safe way to reduce muscle passive force is by applying an external force, for example, through massage. Research shows that 
massage can promote muscle relaxation $[8,9]$, reduce muscle tension [10] and soreness [11, 12], accelerate muscle function recovery [13], and improve athletic performance [14]. Other methods, such as self-myofascial release, exert pressure on the muscles (in this case, using one's own body weight on structures, such as foam rollers) to reduce passive force [15]. However, these methods mentioned above are time-consuming and labor-consuming, and at present, the mechanism of passive muscle force change caused by the applied force is not clear. A simple, labor-saving and noninvasive therapy for the relief of muscle tension and the improvement of flexibility remains an unmet clinical need. Meanwhile, research on the mechanism of this mechanical effect is helpful in providing a clinical basis and supporting medical evidence for its application in rehabilitation treatments.

Ultrasound is a mechanical wave that transmits mechanical energy to deep tissue through a focused transducer or phased array to produce extensive biological and biochemical effects in cells [16-18]. It is increasingly being used as therapeutic tool to promote biological activity—low frequency low-intensity therapeutic ultrasound effectively stimulates tissue healing and regeneration. Research results show that low-intensity pulsed ultrasound can effectively repair bone defects, accelerate bone healing, accelerate regeneration after fracture, and produce other changes in the body, such as reversible decomposition of cross-linked fibers [19]. In addition, ultrasound, through biosynthetic and catabolic processes, can stimulate the production of osteoblasts and chondrocytes, promote the production of growth factors and other signal molecules, and promote osteogenic differentiation [20-23]. These findings 
suggest that therapeutic ultrasound may be beneficial to fields of application such as cell and musculoskeletal tissue engineering and have great potential for tissue repair, regeneration, and regulation of characteristics.

To investigate the effect of ultrasound on the passive force of soleus muscles and require a better understanding of mechanisms of interaction between ultrasound and tissues, we explored the effect of different ultrasound intensity on dynamic muscle characteristics through a stress relaxation experiment, and studied the changes of muscle microstructure by histological analysis. We evaluated the relationship between the macroscopic response of passive muscle force and the microscopic response of passive skeletal muscle force to ultrasonic mechanical stimulation, to study the related mechanical mechanism of passive muscle force change. The aim of this study was to propose a new method of regulating muscle function and provide technical and theoretical guidance for the rehabilitation and regulation of muscle tissue and function.

\section{Material and methods}

\section{The standard linear solid model of biological tissue}

Complex behavior changes are produced in muscle tissue under the influence of external conditions. The behavior of muscle can be described by known material properties and characteristics. When subject to passive muscle force, an object will deform according to the characteristics of its materials composition. If the strain remains constant, the stress within the object will decrease with time. This 
A

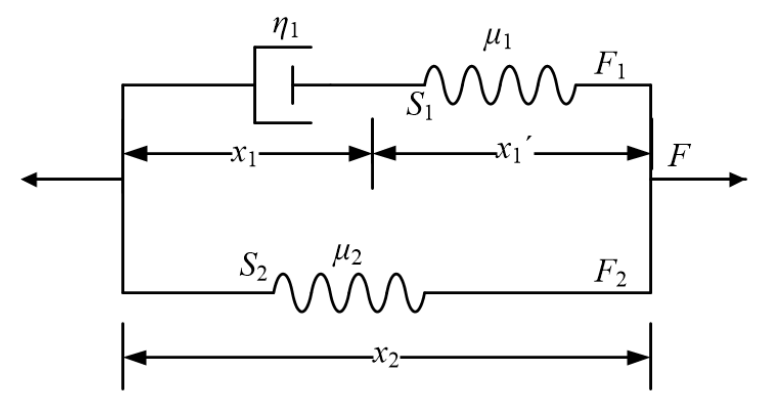

B

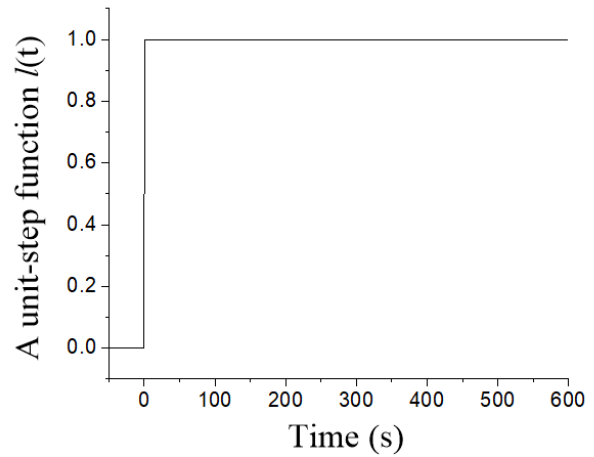

91

Fig. 1. Schematic representation of the standard linear solid model (A) and unit-step function $l(\mathrm{t})(\mathrm{B})$.

cartilage, ligament, and muscle [24]. The physiological function of tissues and organs can be studied with biomechanical mathematical models to understand the complex mechanisms underlying changes in muscle function. The standard linear solid model, the Voigt model, and the Maxwell model are, similarly, composed of linear springs and dampers. Tissue subjected to an external force will deform elastically-the instantaneous elastic properties represented by linear springs - and then stress relaxation will occur - the exponential time-dependent properties represented by the dampers. Compared with the other two models, the standard linear solid model is better for explaining the speed of energy dissipation when tissues are subjected to cyclic forces [25]; therefore, we used the standard linear solid model to explore the changes to the mechanical properties of passive muscle.

The standard linear solid model is shown in Fig. 1A. The model is composed of two branches: one branch-a damper, with viscous coefficient $\eta_{1}$ and displacement represented by $x_{1}$, and spring $S_{1}$, with elasticity coefficient $\mu_{1}$ and displacement 
represented by $x_{1}{ }^{\prime}$, connected in series-is subjected to force $F_{1}$; and the other branch—spring $S_{2}$, with elasticity coefficient $\mu_{2}$ and displacement represented by $x_{2}$-is subjected to force $F_{2}$, when force $F$ is applied to the system. The model satisfies the following relations [25]:

$$
\begin{gathered}
x_{2}=x_{1}+x^{\prime}{ }_{1} \\
F=F_{1}+F_{2} \\
F_{2}=\mu_{2} x_{2} \\
F_{1}=\eta_{1} \dot{x}_{1}=\mu_{1} x^{\prime}{ }_{1}
\end{gathered}
$$

After substituting eq. 3 and eq. 4 into eq. 2 , it can be written eventually as

$$
F+\tau_{\varepsilon} \dot{F}=E_{R}\left(x_{2}+\tau_{\sigma} \dot{x_{2}}\right)
$$

where factor $\tau_{\varepsilon}=\eta_{1} / \mu_{1}$ is the relaxation time of the passive muscle force under the condition of constant strain, which represents the relaxation speed of the passive force, factor $\tau_{\sigma}=\left(\eta_{1} / \mu_{1}\right)\left(1+\mu_{2} / \mu_{1}\right)$ is the relaxation time of strain under constant stress, and factor $E_{R}=\mu_{2}$ is the relaxation modulus of elasticity. As $t \rightarrow \infty$, the dashpot relaxes completely, and the change in passive force is primarily determined by the spring, which is characterized by $E_{R}$.

If the muscle produces an elongation at $t=0$ that is maintained thereafter, the elongation is represented by function $l(t)$, which is shown in Fig. 1B. The relaxation function $F(t)$, is

$$
F(t)=E_{R}\left[1-\left(1-\tau_{\sigma} / \tau_{\varepsilon}\right) \exp \left(-t / t_{\varepsilon}\right)\right] l(t)
$$

where $F(t)$ is the passive force that must be applied in order to produce an elongation $l(t)$. The time factor $\tau_{\varepsilon}$ represents the time required for muscle passive 
force to reach equilibrium. When $\tau_{\varepsilon}$ increases, the rate of stress tends to equilibrium slows.

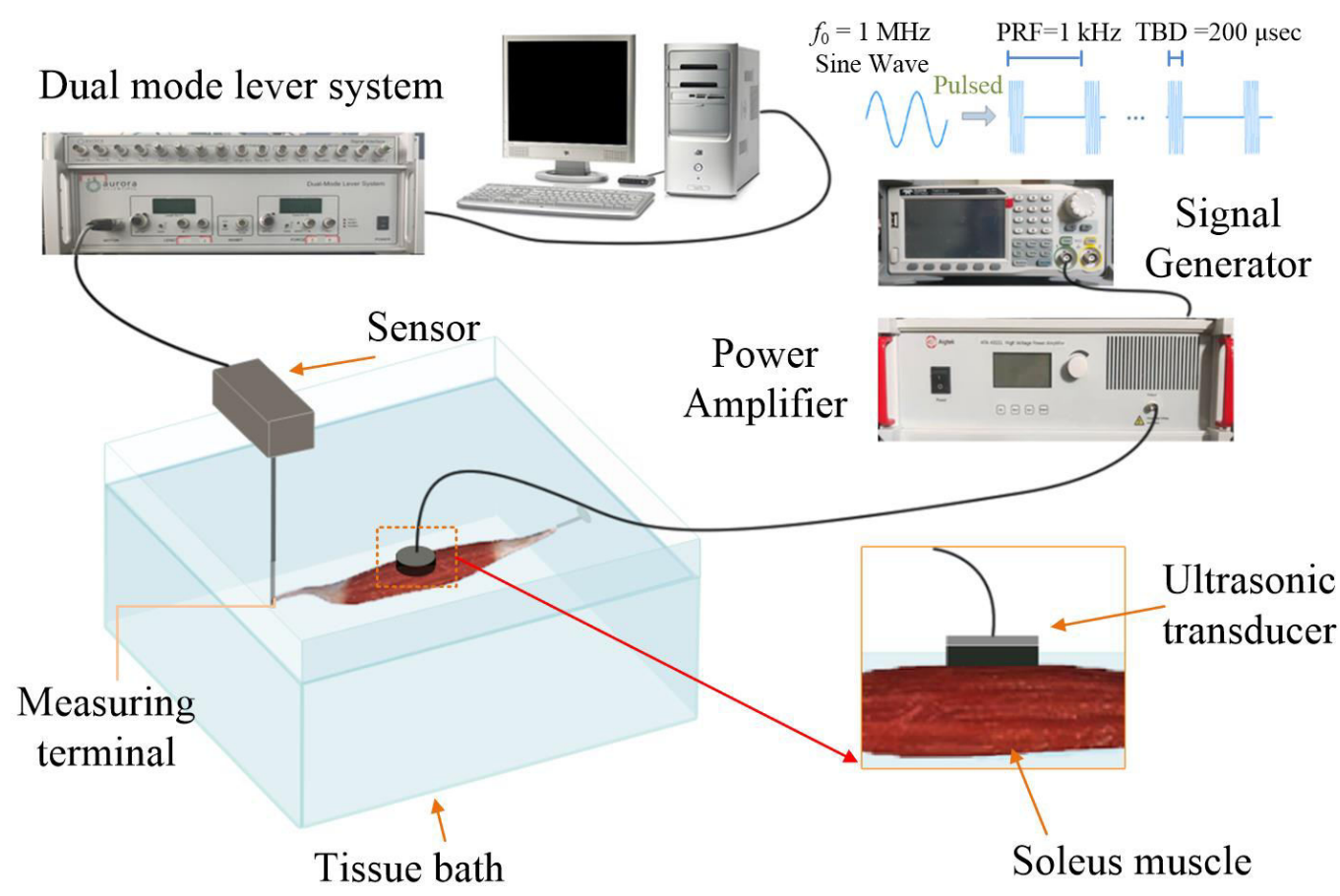

Fig. 2. Schematic diagram of the experimental setup of ultrasound stimulation and the temporal profile of the pulsed ultrasound. A ultrasound pulsed at an ultrasound fundamental frequency $\left(f_{0}\right)$ of $1 \mathrm{MHz}$ for a tone-burst duration (TBD) of 200 $\mu$ s. Such ultrasound pulses were repeated at certain pulse repetition frequency (PRF) $1 \mathrm{kHz}$.

\section{Experiment}

32 male Sprague Dawley rats were obtained from the Laboratory Animal Breeding and Research Center of Xi'an Jiaotong University (Xi'an, China). The experiments were carried out under the condition of constant pre-strain (stress relaxation) and muscle original length. Stress relaxation experiments were carried out on isolated muscle specimens using a whole animal muscle test system (Aurora Scientific Inc, 1305A, Canada). The soleus muscles $(\mathrm{n}=32)$ of male Sprague Dawley rats were used, and the mechanical properties of the soleus muscle were tested with 
and without ultrasound stimulation (ultrasound and control groups, respectively).

The rats were deeply anesthetized by intraperitoneally injecting pentobarbital sodium; the limbs of rats were fixed, the dorsal skin of the hind limbs was removed,

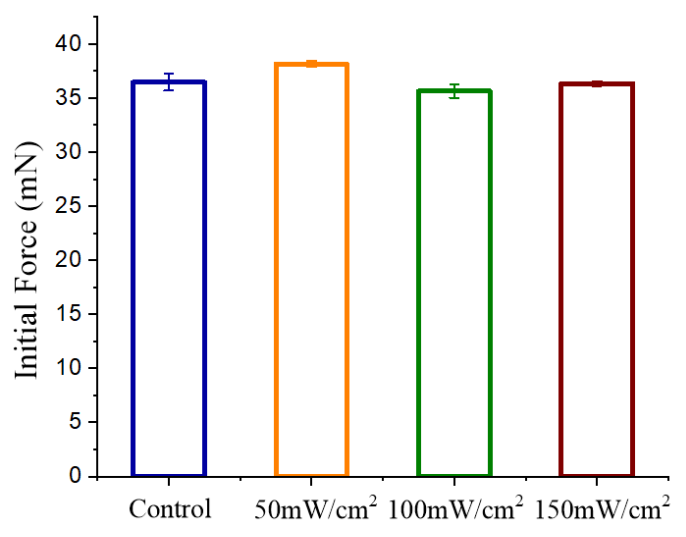

the

Fig. 3. The initial muscle passive force of each group.

tendons on both ends of soleus were ligated with silk thread, and the muscle was fully exposed and gently separated. The soleus muscle was removed wholly intact (with tendons) and placed in Krebs-Henseleit solution (120 mM NaCl, $4.8 \mathrm{mM} \mathrm{KCl}, 20.4$ $\mathrm{mM} \mathrm{NaHCO}_{3}, 10 \mathrm{mM}$ glucose, $1.0 \mathrm{mM}$ pyruvate, $1.2 \mathrm{mM} \mathrm{MgSO}_{4}$, and $1.6 \mathrm{mM} \mathrm{CaCl}_{2}$ ) was kept at approximately $30{ }^{\circ} \mathrm{C}$, and a $95 \% \quad \mathrm{O}_{2} / 5 \% \quad \mathrm{CO}_{2}$ gas mixture was continuously dissolved into the solution.

The whole animal muscle test system for data acquisition consisted of a tissue bath, in which the soleus specimen was fixed, and force sensor, which captured passive muscle force data during the stress relaxation test. The experimental setup and ultrasound temporal waveforms were illustrated in Fig. 2. As presented, the silk thread at one end of the soleus was placed on the bracket inside the tissue bath, and the silk thread at the other end was tied to the lever of the force sensor.

To adjust the muscle to the optimal length, pre-strain was applied to both ends of 
the specimen and remains unchanged. The initial muscle passive force of each group is shown in Fig. 3. An ultrasonic transducer was placed perpendicular to the muscle belly. To ensure that the ultrasonic mechanical energy was transmitted to the muscle tissue with minimum loss, the surface of the transducer was immersed in the liquid and placed in gentle contact with the surface of the muscle. The other end of the transducer was connected to a power amplifier (Aigtek, ATA-43151, China), which amplified the signal from the signal generator (Teledyne LeCroy Inc., T3AFG120, US), the signal was a tone-burst at an ultrasound fundamental frequency $\left(f_{0}\right)$ of $1 \mathrm{MHz}$ for a tone-burst duration (TBD) of $200 \mu$ s (Fig. 2), and data were collected every 2 seconds. In the ultrasound group, three different ultrasound intensities $(50,100$, $150 \mathrm{~mW} / \mathrm{cm}^{2}$ ) were applied to muscle, the ultrasonic transducer was turned on after the experimental platform was set up, and the system began to collect data immediately. After 10 minutes, the transducer was turned off, and data acquisition stopped. In the control group, the transducer remained off during data acquisition (10-minute acquisition time).

To eliminate the interference of muscle stretching on ultrasonic effect, the other 32 soleus muscles received 10 minutes ultrasound irradiation $\left(0,50,100,150 \mathrm{~mW} / \mathrm{cm}^{2}\right)$ in the original length state.

\section{Data fitting}

To study the underlying mechanism of the change of muscle passive force caused by ultrasound stimulation, we used the least squares method to fit the experimental data. Before fitting the data, we modified eq. (6). When $l(t)$ is the unit strain, the 
following can be obtained:

$$
F(t)=E_{R}+\mu_{1} \exp \left(-\mu_{1} t / \eta_{1}\right)
$$

The data were fitted to eq. 7 . We set the minimum value of each set of data as $E_{R}$.

Morphometric analysis of soleus muscle structure
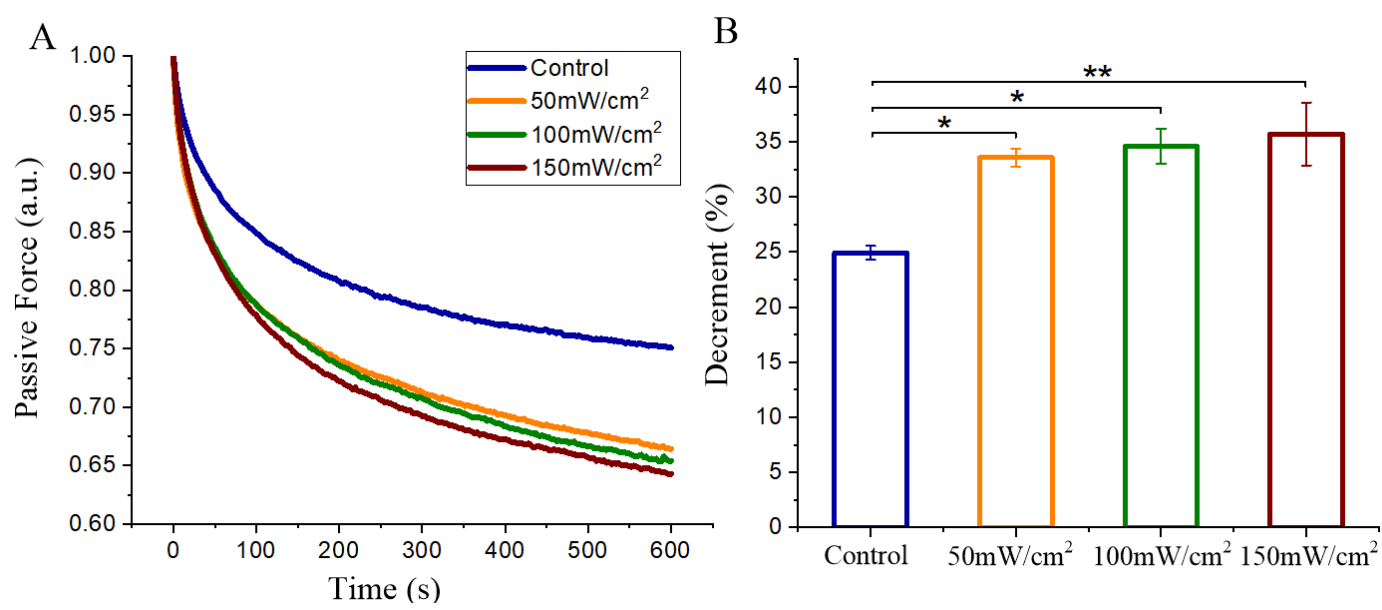

Fig. 4. The effect of three ultrasound intensities on passive force. A. Passive muscle stress relaxation curve showing the time-dependent change profile after normalization. B. The decrement of muscle passive force at ten minutes. Data are expressed as mean $\pm \mathrm{SE}(\mathrm{n}=8$ per group), $* * p<0.01, * p<0.05$.

Hematoxylin and eosin (H\&E) staining was used to qualitatively examine morphological changes in microstructure of soleus muscles. After the experiment, samples were collected and their cross sections were stained with hematoxylin and eosin (H\& E) stain.

\section{Statistical analysis}

Data are expressed as mean \pm standard error (SE) and were analyzed using a one-way analysis of variance and post hoc Tukey multiple comparisons. The viscosity coefficient, elasticity coefficient and passive force decrement of the two groups were compared. A p-value $<0.05(*)$ was considered statistically significant, and a p-value $<0.01(* *)$ was considered a very significant difference. 


\section{Results}

\section{Effect of ultrasonic stimulation on passive muscle force}

This study aimed to identify regimens of noninvasive mechanotherapy applied to
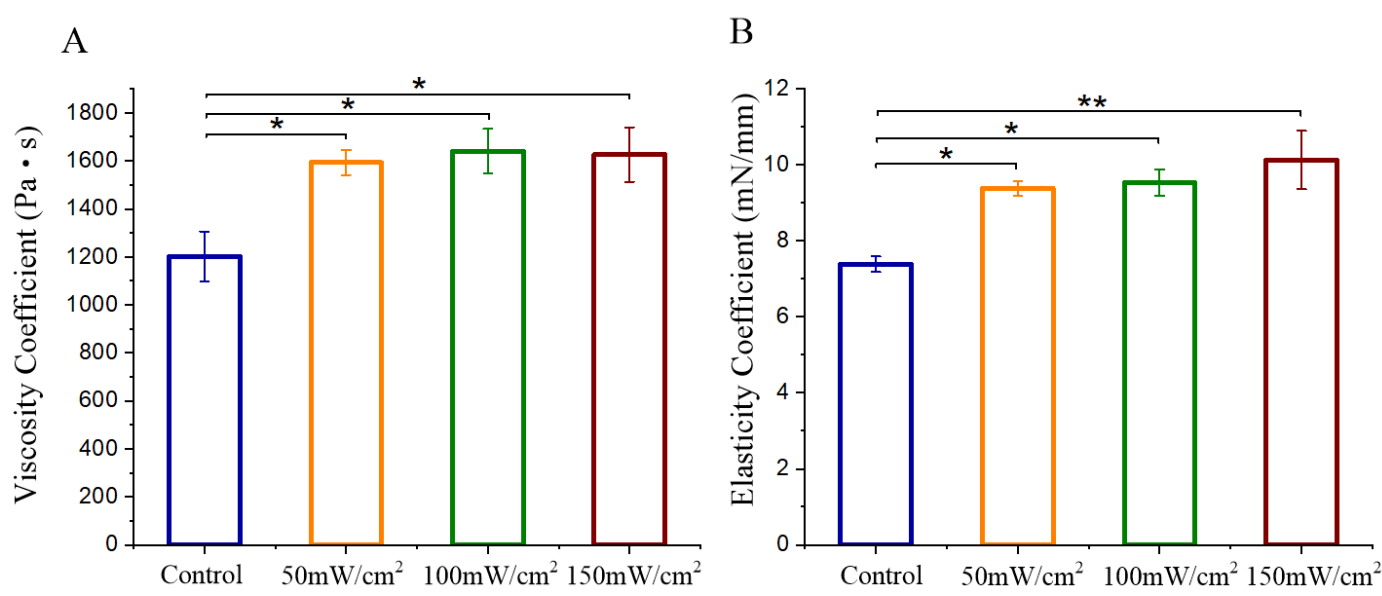

Fig. 5. The effects of ultrasound on the viscosity coefficient, elasticity coefficient. Data are expressed as mean $\pm \operatorname{SE}(n=8$ per group), $* * \mathrm{P}<0.01, * \mathrm{P}<0.05$.

muscle and to probe the functional link between different ultrasonic intensities and stress relaxation. We obtained the relaxation curves through experiment. The results, after normalization, are shown in Fig. 4A. The passive muscle force under all conditions decreased exponentially. In the ultrasound group, three different ultrasonic intensities were applied using ultrasonic transducer, whereas muscles in the control group remained untreated. At the 10th minute, the muscle passive force of the control group had decreased by $24.5 \%$ from the initial state, whereas the muscle passive force of the ultrasound group decreased by $33.6 \%, 34.6 \%$ and $35.7 \%$ under $50 \mathrm{~mW} / \mathrm{cm}^{2}$,

$252100 \mathrm{~mW} / \mathrm{cm}^{2}$ and $150 \mathrm{~mW} / \mathrm{cm}^{2}$, respectively. The statistical results of the passive 253 force decrement are shown in Fig. 4B. As presented, there were significant differences 
254 between ultrasound group and control group, no significant difference between the

255 three different intensities conditions (Fig. 4B, ultrasound intensity $50 \mathrm{~mW} / \mathrm{cm}^{2}$ vs.

256 control: $p=0.006$; ultrasound intensity $100 \mathrm{~mW} / \mathrm{cm}^{2}$ vs. control: $p=0.002$;

257 ultrasound intensity $150 \mathrm{~mW} / \mathrm{cm}^{2}$ vs. control: $\left.p=0.0006.\right)$.

\section{Effect of ultrasonic stimulation on the viscoelasticity of muscle tissue}

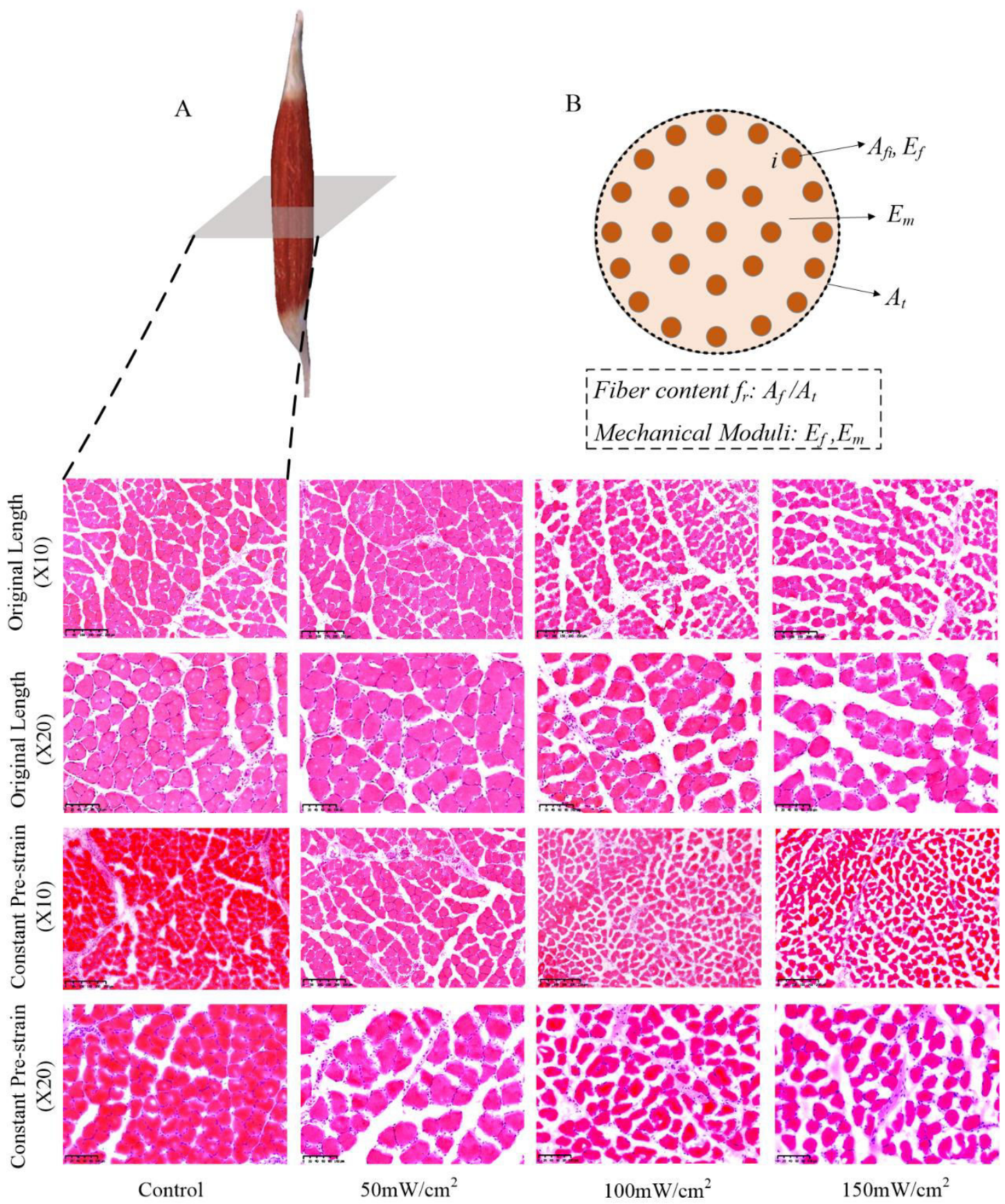


Fig. 6. A. Representative hematoxylin and eosin (H\&E) images of transverse histological sections of the soleus muscle in the original length state and constant pre-strain state. B. The schematic diagram of muscle fibers embedded in a matrix substance. $A_{f}$ is the fibrillar area and $A_{t}$ the total area, $E_{m}, E_{f}$ is the elastic muduli for matrix and fibril, respectively [48].

We made use of experimental relaxation curves of soleus muscles, upon which we identify the elasticity parameters and viscosity parameters characterizing the time-dependent behavior of each soleus muscle. The coefficient of determination $\mathrm{R}^{2}$ of the curve was between 0.95 and 0.99 , and with a mean value 0.97 , which proves

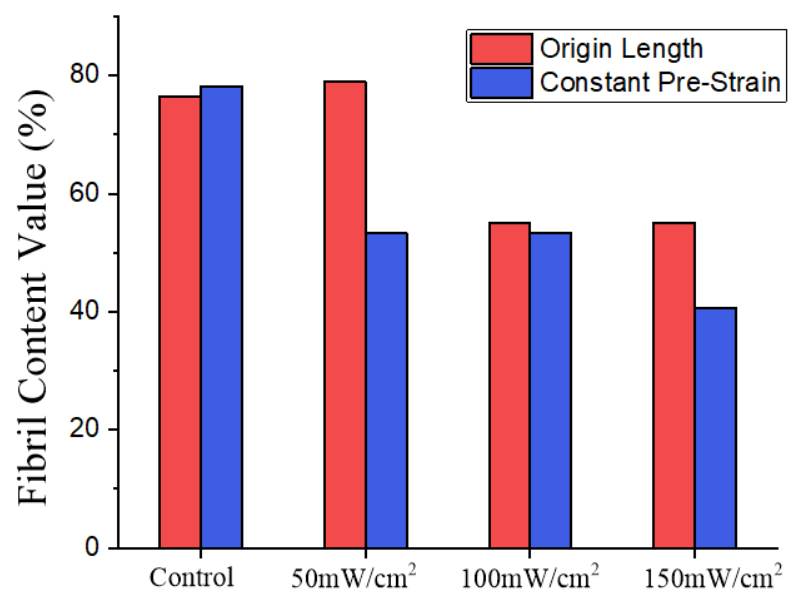

the

Fig. 7. The effect of three different intensities on the the fibril content in the original length state and constant pre-strain state.

reliability of using the standard linear solid model to analyze the experimental data.

The

statistical results of the effect of different ultrasound intensities on viscosity and elasticity coefficient shown in Fig. 5. As presented, the mean viscosity coefficients were 1202.1, 1593.42, 1640.26, $1626.553 \mathrm{~Pa} \cdot \mathrm{s}$ for control group and 50, 100, $150 \mathrm{~mW} / \mathrm{cm}^{2}$, respectively (Fig. $5 \mathrm{~A}$, ultrasound intensity $50 \mathrm{~mW} / \mathrm{cm}^{2}$ vs. control: $p=$ 0.030; ultrasound intensity $100 \mathrm{~mW} / \mathrm{cm}^{2}$ vs. control: $p=0.013$; ultrasound intensity $150 \mathrm{~mW} / \mathrm{cm}^{2}$ vs. control: $p=0.017$.); the mean elasticity coefficients were $7.39,9.38$, 9.53, $10.13 \mathrm{mN} / \mathrm{mm}$ for control group and 50,100,150mW/cm ${ }^{2}$, respectively (Fig. 5B, 
ultrasound intensity $50 \mathrm{~mW} / \mathrm{cm}^{2}$ vs. control: $p=0.017$; ultrasound intensity 100 $\mathrm{mW} / \mathrm{cm}^{2}$ vs. control: $p=0.009$; ultrasound intensity $150 \mathrm{~mW} / \mathrm{cm}^{2}$ vs. control: $p=$ 0.0008.).

\section{Effect of ultrasonic stimulation on microstructure of tissue}

Histological observations (Fig. 6) showed some typical characteristics of soleus muscles. In the transverse section of the soleus muscles, the fibers in the control group were relatively thick and close. In the ultrasound group, with the enhancement of ultrasonic intensity, the space between fibers increased, the fibril content was decreased and the matrix substance increased. Quantitative analysis of immunohistochemistry was performed with Image-pro plus 6.0 using the standard acquisition camera mode $(10 \times, 20 \times$ objective). We calculated the fibril content in the original length state and constant pre-strain state (20x transverse section in Fig. 6), and the results were showed in Fig. 7. In the control group, the fibril content was $78.2 \%$, and as the ultrasonic intensity increased, the fibril contents were $53.4 \%, 53.4 \%$ and $40.6 \%$. When the muscles keep their original length, the fibril content was $76.4 \%$, and as the ultrasonic intensity increased, the fibril contents were $79 \%, 54.6 \%$ and $55.1 \%$.

\section{Discussion}

The objective of this study was to study the influence of low-intensity pulsed ultrasound on the mechanical properties of passive muscle using the stress relaxation 
test and rheological model and to characterize the effects by the changes to viscosity, and microstructure of tissue.

\section{Effect of ultrasonic stimulation on passive muscle}

Anderson et al. used a duration of $250 \mathrm{~s}$ to test muscles from mice [26], Myer et al. used a duration of $300 \mathrm{~s}$ to test muscles from rabbits [27], Van Loocke et al. used a duration of $300 \mathrm{~s}$ to test porcine muscle specimens [28], and Gras et al. used a duration of $840 \mathrm{~s}$ for the sternocleidomastoid muscle from humans [29]. In our study, we used a duration of 10 minutes (600 s) for the stress relaxation tests. force.

The force-time curves that we obtained (Fig. 4A) had similar appearances to those in the literature [26-28, 30]; the force decreased exponentially. As presented (Fig. 4B.), the soleus muscles treated with ultrasound at all three ultrasonic intensities significantly exhibited higher decrement than the untreated control group, with no significant difference between the three ultrasonic intensities. This phenomenon is consistent with conclusions in the literature. Siebert et al. [31-34] reported that the longitudinal muscle force of isolated rat muscles decreases when subjected to unilateral transverse compression. Reinhardt et al. reported that transverse compression forces can be transmitted between muscles and potentially affect the how longitudinal muscle force is generated [35]. The statistical results also proved that ultrasonic mechanical stimulation could accelerate the reduction of muscle passive

\section{Effect of ultrasonic stimulation on the viscoelasticity of muscle tissue}



tissue's relaxation behavior, mathematically described as a function of both elastic and viscous parameters. The functional properties of muscle can usually be quantified by the elastic component of the tissue, and the microstructural changes can be characterized by the viscosity component. Research on the muscle viscoelasticity can better explain the underlying causes of changes to muscle function and underlying 346 pathophysiology. These functional parameters (elasticity and viscosity) can be

determined using rheological models. The standard linear solid model can be used to describe the properties of more complex, uniform, incompressible viscoelastic media. It can not only accurately describe strain creep and recovery processes but can also accurately describe stress relaxation processes. In the current work, we combine the obtained results with the standard linear solid model to extract information of viscoelastic effect of ultrasound on muscle.

In this paper, the viscosity and elasticity value obtained through model fitting was presented. The mean muscle viscosity coefficient and elasticity coefficient after ultrasonic stimulation were significantly greater than those found for the group without ultrasonic stimulation. With the increase of sound intensity, the viscosity coefficients increased by $32.5 \%, 36.4 \%$ and $35.3 \%$ compared with the control group, the elasticity coefficients increased by $27 \%, 29.1 \%$ and $37.1 \%$ compared with the control group, and with no significant difference between three intensities. These results are consistent with those found in the literature suggesting that viscosity and elasticity increase with increasing load [36]. Our findings suggest that the 
viscoelasticity of muscle tissue changes significantly after ultrasound stimulation, which may be the mechanism of muscle force reduction from ultrasound stimulation.

The vibration effect of the ultrasound wave and acoustic radiation force affect the biological transmission medium. The mechanical effect is the primary feature of the ultrasonic wave and exists in an ultrasonic propagation medium regardless of ultrasonic intensity. As a non-invasive treatment, ultrasound can transfer mechanical energy to tissues and produce extensive biological and biochemical effects in cells [16-18], causing changes such as the reversible decomposition of cross-linked fibers [19]. It can stimulate the cells' growth in vitro experiments through anabolism and promote the production of growth factors and other signal molecules [20-23].

This study suggests that the soleus muscle absorbs the energy of sound wave vibration in the experimental process, and heat energy is generated inside the muscle, which increases the muscle temperature, reduces the internal friction between the muscle fibril and the matrix, and changes the viscoelasticity of the muscle.

\section{Effect of ultrasonic stimulation on microstructure of tissue}

Ultrasonic mechanical effect changes the structure of muscle fibers, which may also be the reason for the change of stress relaxation. The fascicles are composed of fibrils immersed in an extra-cellular non-collagenous matrix substance which maintains the muscles' structural stability. The embedding matrix is primarily composed of water that is interspersed with cells and proteoglycans [37]. A series of relaxation processes such as sliding and shearing mechanisms have been observed at the embedding matrix scale. [38, 39]. Relevant studies suggest that the matrix 
substance plays a significant role in the relaxation process.

When the effect of matrix substance on muscle viscoelasticity is considered, the muscle with elastic and viscous properties $E_{f}, E_{m}$ and $\eta_{f}, \eta_{m}$ for the fibrillar and matrix components accordingly. And the viscous and elastic parameters can be defined as follows [40]:

$$
\langle\eta\rangle=\eta_{f} f_{r}+\eta_{m}\left(1-f_{r}\right),\langle E\rangle=E_{f} f_{r}+E_{m}\left(1-f_{r}\right)
$$

where in equation (8), $f_{r}$ is fibril content that defined as the ratio of the area $A_{f}$ covered by fibril (dark circle) over the total fascicle cross sectional area $A_{t}$ (Fig. 6B). We observed microstructure of tissue by H\&E staining. Quantitative analysis of immunohistochemistry was performed with Image-pro plus 6.0 using the standard acquisition camera mode $(10 \times, 20 \times$ objective). We calculated the fibril content obtained from two states with and without pre-strain. Results show that the fibril content decreased as the ultrasonic intensity increased, and when ultrasound reached a certain intensity, the decrease of fibril content was mainly attributed to ultrasound instead of pre-strain.

Relevant studies show that the fibrillar content values in the range between $55 \%$ and $85 \%$ for wet rat tail tendon collagen fibrils [41], and in the range of $60 \%$ for human patellar tendons [42]. With the reported relative fibril content differences of more than $40 \%$ to be attributed to swelling effects [43]. When the fiber expands to a certain extent, the fibril content $f_{r}$ is as low as 35\% [44] and up to more than $60 \%$ $[42,45]$. Moreover, some results show that the greater the water content of the tissue, the more obvious the stress relaxation [46]. The level of hydration of the fibrillar 
content is directly affected by the constitution of the matrix embedding matrix substance, which plays a major role in the overall soft tissue mechanics [47].

The experimental results show that ultrasound can promote stress relaxation, and the muscle stimulated by ultrasound showed higher viscosity, which may be related to the fibril content. The results are consistent with that of Karathanasopoulos [48], in which a minimum viscoelastic modulus value is obtained for the highest fibril content, and a maximum viscosity parameter is obtained for the lowest fibrillar content. That is, the viscosity parameter was negatively correlated with muscle fibrillar content. Moreover, the results shows that low fibrillar contents $f_{r}$ pair to high relaxation moduli values (fiber's elastic modulus loss).

The fibrillar components are in a certain relative density to the embedding matrix substance. Such phenomenological effects maybe arise from inner structural changes, which alter the mechanical properties of the muscles. This study considered that ultrasonic mechanical effect may cause muscle swelling, increase cell matrix substance and water content in muscle, reduce muscle fibril content, resulting in viscoelastic changes and eventually change stress relaxation.

\section{Conclusion}

In the current work, we investigate the effect of ultrasound on the passive force of soleus muscles. We combine the obtained results with the standard linear solid model to extract information of viscoelastic effect of ultrasound on muscle, and calculate muscle fibril content by histological analysis. The results show that the 
ultrasound group has higher decrement of muscle passive force, larger viscosity coefficient, elasticity coefficient and lower fibril content. On the one hand, we hypothesize that the changes of muscle characteristics maybe related to ultrasonic thermal effect, which reduces the internal friction between the muscle fibril and the matrix, and changes the viscoelasticity of the muscle. On the other hand, the change of muscle microstructure maybe another mechanism that changes viscoelasticity and stress relaxation. These results provide an important theoretical basis and a feasible method for monitoring muscle functional characteristics by measuring muscle viscoelasticity.

\section{Abbreviations}

PNF: Proprioceptive neuromuscular facilitation; TBD: tone-burst duration; PRF: pulse repetition frequency; H\&E: Hematoxylin and eosin; SE: standard error.

\section{Acknowledgements}

Not applicable.

\section{Authors' contributions}

Xuebing Yang designed the study, performed experiments, analyzed data, and wrote the manuscript. Xueqing Zhang and Pan Li performed experiments, Jianzhong Guo designed the study and secured funding, Liang Tang and Dean Ta discussed and interpreted results. All authors read, edited, and approved the final manuscript.

\section{Funding}

This work was supported by the National Natural Science Foundation of China [Grant numbers 11727813]; the Fundamental Research Funds for the Central Universities [Grant numbers 
The datasets used and/or analysed during the current study are available from the corresponding author on reasonable request.

\section{Declarations}

Ethics approval and consent to participate

All experiments were conducted with the approval of the Animal Ethical Committee of Shaanxi published by the US National Institutes of Health (NIH publication no. 8523, revised 1996).

\section{Consent for publication}

Not applicable.

\section{Competing interests}

The authors declare that they have no competing interests.

\section{Author details}

Technology, Shaanxi Normal University, Xi'an 710119, China.

${ }^{2}$ Institute of Sports Biology, Shaanxi Normal University, Xi'an 710119, China

${ }^{3}$ Department of Electronic Engineering, Fudan University, Shanghai 200433, China

${ }^{4}$ Human Phenome Institute, Fudan University, Shanghai 201203, China

${ }^{5}$ Key Laboratory of Medical Imaging Computing and Computer Assisted Intervention (MICCAI)

\section{References}


1. Wiltvrouw E, Mahieu N, Danneels L and McNair P. Stretching and Injury Prevention, an Obscure Relationship. Sports Med. 2004; 34: 443-449.

2. Andersen JC. Flexibility in Performance: Foundational Concepts and Practical Issues. Athlet Ther Today. 2006; 3: 9-12

3. Haff G. Roundtable discussion: Flexibility training. J Strength and Cond Res. 2006; 28:64-85.

4. O'Hora J, Cartwright A, Wade CD, Hough AD, Shum GL. Efficacy of static stretching and proprioceptive neuromuscular facilitation stretch on hamstrings length after a single session. J Strength Cond Res. $2011 ; 25(6): 1586-1591$.

5. Fagnani F, Giombini A, Di Cesare A, Pigozzi F, Di Salvo V. The effects of a whole-body vibration program on muscle performance and flexibility in female athletes. Am J Phys Med Rehabil. 2006;85(12):956-962.

6. Gerodimos V, Zafeiridis A, Karatrantou K, Vasilopoulou T, Chanou K, Pispirikou E. The acute effects of different whole-body vibration amplitudes and frequencies on flexibility and vertical jumping performance. J Sci Med Sport. 2010;13(4):438-443.

7. Jacobs PL, Burns P. Acute enhancement of lower-extremity dynamic strength and flexibility with whole-body vibration. J Strength Cond Res. 2009;23(1):51-57.

8. Nordschow $\mathrm{M}$ and Bierman $\mathrm{W}$. The influence of manual massage on muscle relaxation: effect on trunk flexion. J Am Phys Ther Assoc. 1962; 42(10): 653.

9. Wiktorsson-Moller M, Oberg B, Ekstrand J, Gillquist J. Effects of warming up, massage, and stretching on range of motion and muscle strength in the lower extremity. Am J Sports Med. 1983; 11 (4): 249-52.

10. Dubrosky V. Changes in muscle and venous blood flow after massage. Soviet Sports Rev. 1983; 18(3): $134-135$.

11. Tiidus P, Shoemaker J. Effleurage massage, muscle blood flow and long-term post-exercise recovery. Int J 
12. Smith LL, Keating MN, Holbert D, Spratt DJ, McCammon MR, Smith SS, Israel RG. The effects of athlete massage on delayed onset muscle soreness, creatine kinase and neutrophil count: a preliminary report. J Orthop Sports Phys Ther. 1994; 19 (2): 93-99.

13. Starkey AJ. Treatment of ankle sprains by simultaneous use of intermittent compression and ice packs. Am J Sports Med. 1976; 4 (4): 142.

14. Rinder AN, Sutherland CJ. An investigation of the effects of massage on quadriceps performance after exercise fatigue. Complement Ther Nurs Midwifery. 1995; 1(4): 99-102.

15. PM Keys. The effects of myofascial release vs static stretching on hamstrings range of motion. Southern Illinois University Carbondale. 2014.

16. Wu J, Nyborg W. Emerging therapeutic ultrasound. Imperial College Press. 2006.

17. Haar GT. Therapeutic applications of ultrasound. Progr Biophys Mol Biol. 2007; 93(1-3): 111-129.

18. Paliwal S and Mitragotri S. Therapeutic opportunities in biological responses of ultrasound. Ultrasonics. 2008; 48(4): 271-278.

19. Braaten JV, Goss RA and Francis CW. Ultrasound reversibly disaggregates fibrin fibers. Thromb Haemost.

20. Zhou HY and Zhang C. Low-intensity pulsed ultrasound repair in mandibular condylar cartilage injury rabbit model. Archives of Oral Biology. 2019; 104: 60-66.

21. Teoh KH, Whitham R, Wong JF and Hariharan K. The use of low-intensity pulsed ultrasound in treating activity of osteoclasts: an in vitro study. Arch Oral Biol. 2016; 70: 73-78. 

model for the analysis of bone metabolism by ultrasound. Comp Biochem Physiol A Mol Integr Physiol. 2016; 195:26-31.

24. Woo SL, Young EP. Structure and function of tendons and ligaments. 1991. In: Mow VC, Hayes WC, eds. Basic Orthopaedic Biomechanics. New York, NY: Raven Press. 1992244. \& Nerve. 2001; 24(8):1090-1092.

27. Myers BS, Ee CAV, Camacho DLA, Woolley CT, Best TM. On the structural and material properties of

28. Van Loocke M, Lyons CG, Simms CK. Viscoelastic properties of passive skeletal muscle in compression:

30. Bosboom EM, Hesselink MK, Oomens CW, Bouten CV, Drost MR, Baaijens FP. Passive transverse

31. Siebert T, Till $\mathrm{O}$ and Blickhan R. Work partitioning of transversally loaded muscle: experimentation and muscle loading. J Biomech. 2014b; 47: 1822-1828. DOI: 10.1016/j.jbiomech.2014.03.029. 
33. Siebert T, Rode C, Till O, Stutzig N and Blickhan R. Force reduction induced by unidirectional transversal muscle loading is independent of local pressure. J Biomech. 2016; 49: 1156-1161.

34. Siebert T, Stutzig N and Rode C. A hill-type muscle model expansion accounting for effects of varying transverse muscle load. J Biomech. 2018; 66: 57-62.

35. Reinhardt L, Siebert T, Leichsenring K, Blickhan R and Bol M. Intermuscular pressure between synergistic muscles correlates with muscle force. J Exp Biol. 2016; 219: 2311-2319..

36. Gennisson JL, Deffieux T, Macé E, Montaldo G, Fink M, Tanter M. Viscoelastic and anisotropic mechanical properties of in vivo muscle tissue assessed by supersonic shear imaging. Ultrasound in Med \& Biol. 2010; 36(5): 789-801.

37. Screen HRC, Shelton JC, Chhaya VH, Kayser MV, Bader DL, Lee DA. The Influence of Noncollagenous Matrix Components on the Micromechanical Environment of Tendon Fascicles. Ann Biomed Eng. 2005; 33(8), 1090-1099.

38. Screen HRC. Investigating load relaxation mechanics in tendon. J Mech Behav Biomed Mater. 2008; 1(1), $51-58$.

39. Gupta HS, Seto J, Krauss S, Boesecke P, Screen HRC. In situ multi-level analysis of viscoelastic deformation mechanisms in tendon collagen. J Struct Biol. 2010; 169 (2), 183-191.

40. Ganghoffer JF, Laurent C, Maurice G, Rahouadj R, Wang X. Nonlinear viscous behavior of the tendons fascicles from the homogenization of viscoelastic collagen fibers. Eur J Mech- A/Solids. 2016; 59, 265-279.

41. Goh KL, Holmes DF, Lu HY, Richardson S, Kadler KE, Purslow PP, Wess TJ. Ageing changes in the tensile properties of tendons: influence of collagen fibril volume fraction. J Biomech Eng. 2008; 130 (2), 021011.

42. Hansen P, Haraldsson BT, Aagaard P, Kovanen V, Avery NC, Qvortrup K, Larsen JO, Krogsgaard M, Kjaer M, Magnusson SP. Lower strength of the human posterior patellar tendon seems unrelated to mature collagen 
cross-linking and fibril morphology. J Appl Physiol. 2010; 108, 47-52.

43. Robinson KA, Sun M, Barnum CE, Weiss SN, Huegel J, Shetye SS, Lin L, Saez D, Adams SM, Iozzo RV, Soslowsky LJ, Birk DE. Decorin and biglycan are necessary for maintaining collagen fibril structure, fiber realignment, and mechanical properties of mature tendons. Matrix Biol. 2017; 64, 81-93.

44. Svensson RB, Hansen P, Hassenkam T, Haraldsson BT, Aagaard P, Kovanen V, Krogsgaard M, Kjaer M, Magnusson SP. Mechanical properties of human patellar tendon at the hierarchical levels of tendon and fibril. J Appl Physiol. 2012; 112 (3), 419-426.

45. Svensson RB, Hassenkam T, Grant C, Magnusson SP. Tensile properties of human collagen fibrils and fascicles are insensitive to environmental salts. Biophys J. 2010; 99 (12), 4020-4027.

46. Chimich D, Shrive N, Frank C, Marchuk L, Brayet R. Water content alters viscoelastic behaviour of the normal adolescent rabbit medial collateral ligament. J Biomech. 1992; 25(8), 831-837.

47. Ansorge HL, Adams S, Birk DE, Soslowsky LJ. Mechanical, compositional, and structural properties of the post-natal mouse achilles tendon. Ann Biomed Eng. 2010; 39 (7), 1904-1913.

48. Karathanasopoulos N, Ganghoffer JF. Exploiting Viscoelastic Experimental Observations and Numerical Simulations to Infer Biomimetic Artificial Tendon Fiber Designs. Front Bioeng Biotech. 2019; 7, 85. 